TITLE:

\title{
Clinical evaluation of time-of-flight MR angiography with sparse undersampling and iterative reconstruction for cerebral aneurysms
}

\section{$\operatorname{AUTHOR}(S)$ :}

Fushimi, Yasutaka; Okada, Tomohisa; Kikuchi, Takayuki; Yamamoto, Akira; Okada, Tsutomu; Yamamoto, Takayuki; Schmidt, Michaela; Yoshida, Kazumichi; Miyamoto, Susumu; Togashi, Kaori

\section{CITATION:}

Fushimi, Yasutaka ...[et al]. Clinical evaluation of time-of-flight MR angiography with sparse undersampling and iterative reconstruction for cerebral aneurysms. NMR in biomedicine 2017, 30(11): e3774.

\section{ISSUE DATE:}

2017-11

URL:

http://hdl.handle.net/2433/235677

\section{RIGHT:}

This is the peer reviewed version of the following article: YYasutaka Fushimi etc. Clinical evaluation of time - of - flight MR angiography with sparse undersampling and iterative reconstruction for cerebral aneurysms. NMR in biomedicine, 30(11) e3774], which has been published in final form at https://doi.org/10.1002/nbm.3774. This article may be used for non-commercial purposes in accordance with Wiley Terms and Conditions for Use of Self-Archived Versions.; The fulltext file will be made open to the public on 16 October 2018 in accordance with publisher's 'Terms and Conditions for Self-Archiving'; この論文は出版社版でありません。引用の際には出版社版をご確認ご利用ください。; This is not the published version. Please cite only the published version. 
Title

Clinical Evaluation of Time-of-Flight MR Angiography with Sparse Undersampling and Iterative Reconstruction for Cerebral Aneurysms.

Running Title

Compressed Sensed TOF-MRA for Cerebral Aneurysms

Authors

Yasutaka Fushimi, MD, PhD ${ }^{1)}$, Tomohisa Okada, $\mathrm{MD}, \mathrm{PhD}^{2)}$,

Takayuki Kikuchi, MD, PhD ${ }^{3)}$, Akira Yamamoto, $\mathrm{MD}, \mathbf{P h D}{ }^{1)}$,

Tsutomu Okada, MD, PhD ${ }^{1)}$, Takayuki Yamamoto, MD ${ }^{1)}$, Michaela Schmidt ${ }^{4)}$,

Kazumichi Yoshida, MD, PhD ${ }^{3)}$, Susumu Miyamoto, $\mathbf{M D}, \mathbf{P h D}^{3 \text { ), }}$

and Kaori Togashi, $\mathrm{MD}, \mathrm{PhD}^{1 \text { ). }}$

yfushimi@kuhp.kyoto-u.ac.jp

tomokada@kuhp.kyoto-u.ac.jp

tkik@kuhp.kyoto-u.ac.jp

yakira@kuhp.kyoto-u.ac.jp 
tokadat@kuhp.kyoto-u.ac.jp

takatin@kuhp.kyoto-u.ac.jp

michaela.schmidt@siemens.com

kazuy@kuhp.kyoto-u.ac.jp

miy@kuhp.kyoto-u.ac.jp

ktogashi@kuhp.kyoto-u.ac.jp

1) Department of Diagnostic Imaging and Nuclear Medicine, Kyoto University Graduate

School of Medicine. 54 Shogoin Kawaharacho, Sakyoku, Kyoto, JAPAN 6068507

2) Human Brain Research Center, Kyoto University Graduate School of Medicine. 54

Shogoin Kawaharacho, Sakyoku, Kyoto, JAPAN 6068507

3) Department of Neurosurgery, Kyoto University Graduate School of Medicine. 54

Shogoin Kawaharacho, Sakyoku, Kyoto, JAPAN 6068507

4) Diagnostic Imaging, Siemens Healthcare GmbH, Erlangen, Germany 91052

\section{Corresponding author}

Yasutaka Fushimi, MD, PhD

Department of Diagnostic Imaging and Nuclear Medicine 
Graduate School of Medicine, Kyoto University

54 Shogoinkawaharacho, Sakyo, Kyoto, 606-8507, Japan

Office phone: +81-75-751-3760

Office FAX: +81-75-771-9709

\section{Any funding information}

This work was partly supported by Grant-in-Aid for Scientific Research on

Innovative Areas “Initiative for High-Dimensional Data-Driven Science through

Deepening of Sparse Modeling”, MEXT grant numbers 25120002, 25120008.

\section{Acknowledgments}

We are grateful to Dr. Aurelien Stalder, Siemens Healthcare, and Dr. Yutaka

Natsuaki for their help in the development of the Sparse TOF sequence. We are also

grateful to Mr. Katsutoshi Murata and Mr. Yuta Urushibata, Siemens Healthcare K.K., Japan for their continuous help in MR acquisition. 


\section{Manuscript Title}

Clinical Evaluation of Time-of-Flight MR Angiography with Sparse Undersampling and Iterative Reconstruction for Cerebral Aneurysms.

3630 words

Research Article 


\section{ABSTRACT}

Compressed sensing (CS) MRI has just been introduced to research areas as an innovative approach to accelerate MRI. CS is expected to achieve higher k-space undersampling by exploiting the underlying sparsity in an appropriate transform domain. MR angiography (MRA) provides high spatial resolution information of arteries; however, relatively long acquisition time is necessary to cover a wide volume. Reduction of acquisition time by compressed sensing for time-of-flight (TOF) MR angiography (Sparse-TOF) is beneficial in clinical examinations, therefore, clinical validity of Sparse-TOF needs to be investigated. The aim of this study was to compare diagnostic capability of time-of-flight (TOF) MRA between parallel imaging (PI)-TOF with acceleration factor of 3 (annotated as $3 \times$ ) and Sparse-TOF $(3 \times$ and $5 \times$ ) in patients with cerebral aneurysms. PI-TOF $(3 \times)$ and Sparse-TOF (3× and $5 \times$ ) imaging were performed in 20 patients using a 3T-MRI system. Aneurysms in PI-TOF (3×) and Sparse-TOF ( $3 \times$ and $5 \times$ ) were blindly rated as visible or scarcely visible by neuroradiologists. The neck, height and width of aneurysms were also measured. Twenty-six aneurysms were visualized and rated as visible in PI-TOF (3×) and SparseTOF ( $3 \times$ and $5 \times$ ) with excellent agreement between two raters. No significant differences were found in measured neck, height and width of aneurysms among them. 
Sparse-TOF ( $3 \times$ and $5 \times)$ were acquired and reconstructed within 6 minutes, and

cerebral aneurysms were visible in both of them with equivalent quality to PI-TOF $(3 \times)$.

Sparse-TOF $(5 \times)$ is a good alternative to PI-TOF $(3 \times)$ to visualize cerebral aneurysms.

\section{Key words}

Compressed sensing; cerebral aneurysm; parallel imaging; time-of-flight MR

angiography

\section{Abbreviations}

CS, compressed sensing;

PI, parallel imaging;

G-factor, geometry factor;

GRASP, golden-angle radial sparse parallel imaging;

CE, contrast-enhanced;

CNR, contrast-to-noise ratio;

TOF, time-of-flight;

GRAPPA, GeneRalized Autocalibrating Partial Parallel Acquisition; 
mFISTA, Modified Fast Iterative Shrinkage-Thresholding Algorithm;

ISTA, Iterative Shrinkage Thresholding Algorithm;

FISTA, Fast Iterative Shrinkage Thresholding Algorithm;

NESTA, Nesterov Algorithm; 


\section{Introduction}

Compressed sensing (CS) Magnetic Resonance Imaging (MRI) has been introduced to pre-clinical research areas as an innovative sparse recovery framework that supports $k$-space undersampling for accelerated image acquisition. (1). Parallel imaging (PI) is now routinely used as $k$-space undersampling method by reducing the number of phase-encoding steps. However, the acceleration factor of PI is usually not higher than 2 or 3 (annotated as $2 \times$ or $3 \times$ ) in non-contrast MRI, because further reduction of phase-encoding steps will cause substantial increase in the noise associated with the geometry factor (g-factor) (2-4). On the contrary, CS is expected to achieve higher $k$-space undersampling by exploiting the underlying sparsity in an appropriate transform domain (1).

CS is advantageous in time-resolved dynamic MRI because of its temporal sparsity, therefore, CS on MRI has been applied to 4D imaging, such as cardiac cine imaging, by using Cartesian (5) and non-Cartesian sparse sampling (6,7). Recently, high-temporal-resolution imaging called Golden-angle radial sparse parallel imaging (GRASP) has been realized by combining CS, PI, and golden-angle radial sampling. GRASP was applied for contrast-enhanced dynamic pituitary MRI (8) and freebreathing contrast-enhanced (CE) dynamic abdominal MRI (9). Sparse MRI approaches 
work very well for CE-MRA because bright signals are sparsified within a low background signal with high contrast-to-noise ratio (CNR), which matches the basic assumptions of CS. $(10,11)$. It also works well for non-contrast time-of-flight (TOF) MRA due to sparsity in k-space (12-16).

TOF-MRA provides high spatial resolution information of arteries; however, relatively long acquisition time is necessary to cover a wide volume. Recently, SparseTOF has been introduced as an undersampling image acquisition with iterative reconstruction $(17,18)$. Reduction of acquisition time by Sparse-TOF is beneficial in clinical examinations, therefore, clinical validity of Sparse-TOF needs to be investigated.

In this study, we hypothesized that Sparse-TOF will be comparable in clinical diagnosis to PI-TOF imaging even with a higher acceleration factor. Diagnostic capability was compared between Sparse-TOF $(3 \times$ and $5 \times$ ) and PI TOF $(3 \times)$ in patients with cerebral aneurysms.

\section{Material and Methods}

This prospective study was approved by the local institutional review board.

Twenty consecutive patients who were admitted for cerebral aneurysms in our hospital 
from January 2015 till October 2015 were enrolled in this study. Written informed consent was obtained from patients with cerebral aneurysms. Conventional angiography was performed for each patient, and cerebral aneurysms were diagnosed by conventional angiography as the gold standard. Conventional angiography was not used for comparison between PI-TOF 3×, Sparse-TOF 3× and Sparse-TOF 5×.

\section{Patients}

Twenty patients (age, $62.8 \pm 12.9$ (38 -78) years; 3 males and 17 females)

underwent non-contrast-enhanced Sparse-TOF (based on a prototype sequence) ( $3 \times$ and 5×), and these data were compared with non-contrast-enhanced PI-TOF (GRAPPA, GeneRalized Autocalibrating Partial Parallel Acquisition, 3×). All images were obtained with a 3T-MRI scanner (MAGNETOM Skyra VD13A, Siemens Healthcare GmbH, Erlangen, Germany) by using a 32-channel head coil.

PI-TOF

Imaging parameters of PI-TOF were as follows: TR/TE, 20/3.69 msec; flip angle, 18 degrees; FOV, $202.5 \times 240 \mathrm{~mm}^{2}$; matrix, $270 \times 320$; slice thickness, $0.38 \mathrm{~mm}$; number of slabs, 4; phase-partial-Fourier factor, 7/8; slice-partial-Fourier factor, 7/8; and GRAPPA factor, 3; integrated reference scan. The images were automatically 
interpolated to $540 \times 640$ matrix, and resolution was $0.38 \times 0.38 \mathrm{~mm}^{2}$. Scan time was 4:07 min.

Sparse-TOF

The imaging parameters of Sparse-TOF were as follows: TR/TE, 20/3.69 msec; flip angle, 18 degrees, FOV, $202 \times 220 \mathrm{~mm}^{2}$; matrix, $264 \times 288$; slice thickness, 0.38 mm; slice resolution, 50 \%; slices per slab 48; number of slabs, 4, $16.7 \%$ slice oversampling; integrated reference scan. The images were interpolated to $528 \times 576$ matrix, and the resolution was $0.38 \times 0.38 \mathrm{~mm}^{2}$.

Sampling pattern in $k$-space was designed based on the variable-density Poisson disc pattern in the ky-kz phase-encoding plane of a regular Cartesian grid (Figure 1), and the re-ordering was centric-out. The center part of k-space was fully sampled. Sparse-TOF data was reconstructed using a non-linear iterative SENSE-based prototype algorithm with a constraint to enforce sparsity (10). Acceleration factor of Sparse-TOF was set as $3 \times$ and $5 \times$, respectively. Scan time was 3:52 and 2:43 min, respectively. 
Specifically, images were reconstructed by solving the following minimization problem with a Modified Fast Iterative Shrinkage-Thresholding Algorithm (mFISTA) by using L1 wavelet regularization in the phase encoding direction (10),

$$
\min _{x}\left\{\frac{1}{2}\left\|\sum_{j=1}^{N}\left(y_{i}-F_{u} S_{j} x\right)\right\| \frac{2}{2}+\lambda\left\|W_{x}\right\|_{1}\right\}
$$

where $x$ is the image to reconstruct, $y_{j}$ and $S_{j}$ are the k-space data and coil sensitivity for the j-th coil element, $F_{u}$ is the Fourier undersampling operator, $W$ is the redundant Haar wavelet transform, and $\lambda$ is the normalized regularization weighting factor. The coil sensitivity maps are estimated by division of the individual coil images with a sum-ofsquares image of all coils. Reconstruction of Sparse-TOF $3 \times$ and $5 \times$ was conducted as on-line reconstruction function. The data was not transferred to any other workstation. Undersampled data were seamlessly reconstructed on a standard reconstruction system (processor, Intel Xeon CPU E5-1620 3.60 GHz; installed memory, 32.0 GB; operating system, Windows 7 Ultimate, 64-bit), using 10 iterations with a regularization factor of 0.008. Total reconstruction was finished within 6 minutes.

To elucidate the effect of reconstruction, sharpness at the aneurysm edge was calculated (19) in one representative case of Sparse-TOF 3× and Sparse-TOF 5× reconstructed with 1, 2, 3, 4, 5, 10, 15, 20, 30, 40 and 50 iterations as well as PI TOF $3 \times$. Sharpness was defined as the distance between $20 \%$ and $80 \%$ of maximum signal 
intensity for the Gaussian-fitted line profile of the aneurysm and neighboring structure on the source image of Sparse-TOF 3× and Sparse-TOF $5 \times$ reconstructed with 1 to 50 iterations 1, 2, 3, 4, 5, 10, 15, 20, 30, 40 and 50 iterations as well as PI TOF $3 \times$, calculated using ImageJ software (http://imagej.nih.gov/ij/index.html).

Evaluation

During the anonymization step, PI-TOF and Sparse-TOFs were saved as different research IDs so that the name of image sequence was made blind to the readers. Two neuroradiologists (_._., 21 years of experience, _._., 10 years of experience) blindly evaluated the source images of PI-TOF and Sparse-TOFs by using AQnet (TeraRecon Inc., Foster City, CA). AQnet provided neuroradiologists arbitrary planes of multi-planar reconstruction images, maximum intensity projection (MIP) images and volume rendered images. The two neuroradiologists read the cases without any prior information of the number and the location of aneurysms. Visualization of aneurysms were graded as $2=$ visible (definitely present) or $1=$ scarcely visible (inconclusive). In case aneurysms were not detected by neuroradiologists, those aneurysms on the corresponding imaging sequence were graded as $0=$ invisible. 
The neck, the height and the width of the aneurysms were measured by another neuroradiologist (_._., 27 years of experience) with consensus by the other two neuroradiologists who rated the grades of the aneurysms.

Statistical analysis

All analyses were performed by using MedCalc Statistical Software version 16.4.3 (MedCalc Software bvba, Ostend, Belgium; https://www.medcalc.org; 2016). Inter-rater agreement statistic (Kappa) was analyzed for grades of aneurysms between PI-TOF $3 \times$, Sparse-TOF $3 \times$ and Sparse-TOF $5 \times$.

The neck, the height and the width of the aneurysms among the 3 TOF images were analyzed by One-way analysis of variance. A $P$ value $<0.05$ was considered statistically significant. Inter-class coefficients were also analyzed for the neck, the height and the width of the aneurysms among the 3 TOF images.

\section{Results}

Patients

Twenty-six aneurysms were finally diagnosed by conventional angiography (Table 1). Two aneurysms were found in 6 patients. 
Examples of Image Reconstruction on Sparse-TOF

Representative images of Sparse-TOF 3× and 5× are shown in Figure 2. There are some aliasing artifacts and blurring artifacts in the source images of Sparse-TOF when only 1 iteration was conducted. With 10 iterations, the images with reduced artifacts are created. MIP images of representative cases are shown in Figure 3 and 4.

Sharpness at the aneurysm edge of one representative case of Sparse-TOF $3 \times$ and Sparse-TOF 5× reconstructed with 1, 2, 3, 4, 5, 10, 15, 20, 30, 40 and 50 iterations as well as PI TOF $3 \times$ is shown in Figure 5 . Edge sharpness of Sparse-TOF $3 \times$ and Sparse-TOF 5× increased rapidly between 1 and 10 iterations; however, it did not increase any more after 10 iterations.

\section{Evaluation}

All aneurysms with all 3 imaging protocols were graded as 2 by the neuroradiologists. Kappa value of inter-rater agreement was 1.0 in PI-TOF, Sparse-TOF $3 \times$ and Sparse-TOF $5 \times$.

Measured neck, height and width of all aneurysms are shown in Table 2. There were no significant differences in the measured data among PI-TOF $3 \times$, Sparse-TOF $3 \times$ 
and Sparse-TOF 5× (neck, $\mathrm{P}=0.988$; width, $\mathrm{P}=0.988$; and height, $\mathrm{P}=0.977$ ). Interclass coefficient of the neck, the height and the width of the aneurysms between PI-TOF $3 \times$, Sparse-TOF $3 \times$ and Sparse-TOF $5 \times$ was very good, and the values were as follows: neck, 0.9572, height, 0.9787; and width, 0.9747 .

\section{Discussion}

All aneurysms were recognized by neuroradiologists equally with no differences of confidence in PI-TOF and Sparse-TOFs in this study. CS is a novel algorithm that uses a prior knowledge of underlying sparsity in the images, and more acceleration in undersampling is expected compared with conventional PI. The image characteristics may be different between PI-TOF and Sparse-TOFs, therefore, clinical relevance of Sparse-TOFs should be explored. The detection of aneurysm is especially important in TOF MRA.

TOF MRA with undersampling acquisition needs to provide neuroradiologists with well discernable images of a cerebral aneurysm larger than $7 \mathrm{~mm}$ in diameter, because unruptured aneurysms larger than $7 \mathrm{~mm}$ are associated with an increased risk of rupture (20-22). On the other hand, the aneurysms smaller than $5 \mathrm{~mm}$ in diameter tend to have a low risk of rupture $(20,23)$, therefore, neuroradiologists should not overlook any 
aneurysm larger than $7 \mathrm{~mm}$ in diameter. The average size of aneurysms was about 7.3

mm in this study, therefore, most of aneurysms should not be overlooked in this study.

In this context, even Sparse-TOF $5 \times$ is considered acceptable in clinical practice, because no cerebral aneurysms were overlooked by the neuroradiologists, and no difference was observed in the visualization scores.

The reconstructed images in compressed sensing vary according to the undersampling rate and pattern in the $k$-space (1), the algorithm of compressed sensing, such as Iterative Shrinkage Thresholding Algorithm (ISTA), Fast ISTA (FISTA) (24) and Nesterov Algorithm (NESTA) (25), and the parameters of compressed sensing, such as $\lambda$ and number of iterations (13). All the aneurysms were graded as visible without any difference in size among PI-TOF and Sparse-TOFs. Therefore, the present parameters of image acquisition and reconstruction are recognized as sufficient at least for visualization of cerebral aneurysms of sizes greater than $7.3 \mathrm{~mm}$.

CS application for non-contrast TOF-MRA has been done in previous papers. Retrospective approach using $100 \%$ sampled raw data of TOF-MRA was feasible to assess the reconstructed images with various undersampling rate and various CS reconstruction parameters of NESTA algorithm (13). Another previous study of noncontrast TOF-MRA adopted CS algorithm of mFISTA with prospective undersampling, 
and various undersampling rates were compared in healthy volunteers (12). Sparse-TOF can achieve better image quality relative to PI TOF at higher acceleration factors (12). The undersampling rate of MR angiography in this study is described without considering reference lines at the k-space center. The net acceleration factor taking account of reference lines at the k-space center was 2.4 for PI-TOF 3×, 2.6 for SparseTOF 3×, and 3.8 for Sparse-TOF 5×. Phase-partial-Fourier factor of 7/8 and slicepartial-Fourier factor of 7/8 were applied for PI-TOF only.

This study is the first study of clinical validation for cerebral aneurysm detection on prospectively acquired CS MRA sequence; however, there are several limitations in this study. A relatively small number of patients with cerebral aneurysms was enrolled in this study. True positive cases of cerebral aneurysms were evaluated in this study, therefore, in order to demonstrate clinical validity of CS MRA, it should be conducted for a larger population with or without vascular lesions. Sensitivity and specificity of CS MRA in detection of cerebral aneurysms should be assessed in further studies. An inter-rater agreement of 1.0 was achieved for visualization of aneurysms in this study. The average size of aneurysms was about $7.3 \mathrm{~mm}$ in this study, therefore, most of aneurysms should not be overlooked in this study. Both phase-partial-Fourier and slice-partial-Fourier which were only applied for PI-TOF might decrease SNR, 
therefore, theoretical SNR of PI-TOF will be lower than that of Sparse-TOF with the same undersampling rate. Finally, GRAPPA without regularization was used for image reconstruction of PI-TOF, on the other hand, L1 regularized SENSE reconstruction was used for image reconstruction of Sparse-TOF. GRAPPA with regularization was not available in our institute, however, if available, PI-TOF reconstructed with regularized GRAPPA should be compared with Sparse-TOF.

In conclusion, Sparse-TOF $3 \times$ and $5 \times$ were acquired and reconstructed with clinically acceptable time, and cerebral aneurysms were visible in both Sparse-TOF 3× and $5 \times$ with equivalent quality to PI-TOF. 


\section{References}

1. Lustig M, Donoho D, Pauly JM. Sparse MRI: The application of compressed sensing for rapid MR imaging. Magn Reson Med. 2007;58:1182-1195.

2. Pruessmann KP, Weiger M, Scheidegger MB, Boesiger P. SENSE: sensitivity encoding for fast MRI. Magn Reson Med. 1999;42:952-962.

3. Griswold MA, Jakob PM, Heidemann RM, Nittka M, Jellus V, Wang J, Kiefer B, Haase A. Generalized autocalibrating partially parallel acquisitions (GRAPPA). Magn Reson Med. 2002;47:1202-1210.

4. Fenchel M, Nael K, Seeger A, Kramer U, Saleh R, Miller S. Whole-body magnetic resonance angiography at 3.0 Tesla. Eur Radiol. 2008;18:1473-1483.

5. Wetzl J, Lugauer F, Schmidt M, Maier A, Hornegger J, Forman C. Free-Breathing, Self-Navigated Isotropic 3-D CINE Imaging of the Whole Heart Using Cartesian Sampling. Paper presented at: Proceedings of the 24th International Society for Magnetic Resonance in Medicine2016; Singapore.

6. Usman M, Atkinson D, Odille F, Kolbitsch C, Vaillant G, Schaeffter T, Batchelor PG, Prieto C. Motion corrected compressed sensing for free-breathing dynamic cardiac MRI. Magn Reson Med. 2013;70:504-516.

7. Nam S, Hong SN, Akcakaya M, Kwak Y, Goddu B, Kissinger KV, Manning WJ, Tarokh V, Nezafat R. Compressed sensing reconstruction for undersampled breathhold radial cine imaging with auxiliary free-breathing data. J Magn Reson Imaging. 2014;39:179-188.

8. Rossi Espagnet MC, Bangiyev L, Haber M, Block KT, Babb J, Ruggiero V, Boada F, Gonen O, Fatterpekar GM. High-Resolution DCE-MRI of the Pituitary Gland Using Radial k-Space Acquisition with Compressed Sensing Reconstruction. AJNR Am J Neuroradiol. 2015;36:1444-1449.

9. Chandarana H, Block TK, Ream J, Mikheev A, Sigal SH, Otazo R, Rusinek H. Estimating liver perfusion from free-breathing continuously acquired dynamic gadolinium-ethoxybenzyl-diethylenetriamine pentaacetic acid-enhanced acquisition with compressed sensing reconstruction. Invest Radiol. 2015;50:88-94.

10. Stalder AF, Schmidt M, Quick HH, Schlamann M, Maderwald S, Schmitt P, Wang Q, Nadar MS, Zenge MO. Highly undersampled contrast-enhanced MRA with iterative reconstruction: Integration in a clinical setting. Magn Reson Med. 2015;74:1652-1660.

11. Rapacchi S, Han F, Natsuaki Y, Kroeker R, Plotnik A, Lehrman E, Sayre J, Laub G, Finn JP, Hu P. High spatial and temporal resolution dynamic contrast-enhanced 
magnetic resonance angiography using compressed sensing with magnitude image subtraction. Magn Reson Med. 2014;71:1771-1783.

12. Yamamoto T, Fujimoto K, Okada T, Fushimi Y, Stalder AF, Natsuaki Y, Schmidt M, Togashi K. Time-of-Flight Magnetic Resonance Angiography With Sparse Undersampling and Iterative Reconstruction: Comparison With Conventional Parallel Imaging for Accelerated Imaging. Invest Radiol. 2016;51:372-378.

13. Fushimi Y, Fujimoto K, Okada T, Yamamoto A, Tanaka T, Kikuchi T, Miyamoto S, Togashi K. Compressed Sensing 3-Dimensional Time-of-Flight Magnetic Resonance Angiography for Cerebral Aneurysms: Optimization and Evaluation. Invest Radiol. 2016;51:228-235.

14. Cukur T, Lustig M, Nishimura DG. Improving non-contrast-enhanced steady-state free precession angiography with compressed sensing. Magn Reson Med. 2009;61:1122-1131.

15. Cukur T, Lustig M, Saritas EU, Nishimura DG. Signal compensation and compressed sensing for magnetization-prepared MR angiography. IEEE Trans Med Imaging. 2011;30:1017-1027.

16. Hutter J, Grimm R, Forman C, Hornegger J, Schmitt P. Highly undersampled peripheral Time-of-Flight magnetic resonance angiography: optimized data acquisition and iterative image reconstruction. MAGMA. 2015;28:437-446.

17. Natsuaki Y, Bi X, Zenge MO, Speier P, Schmitt P, Laub G. Time-Of-Flight with Sparse undersampling (TOFu): towards practical MR applications of the Compressed Sensing. Paper presented at: Proceedings of the 22nd International Society for Magnetic Resonance in Medicine2014; Milan, Italy.

18. Stalder AF, Natsuaki Y, Schmidt M, Bi X, Zenge MO, Nadar M, Speier P, Schmitt P, Laub G. Accelerating TOF MRA in Clinical Practice using Sparse MRI with Variable Poisson Density Sampling. Paper presented at: Proceedings of the 23rd International Society for Magnetic Resonance in Medicine2015; Toronto, Canada.

19. Kramer JH, Arnoldi E, Francois CJ, Wentland AL, Nikolaou K, Wintersperger BJ, Grist TM. Dynamic and static magnetic resonance angiography of the supra-aortic vessels at $3.0 \mathrm{~T}$ : intraindividual comparison of gadobutrol, gadobenate dimeglumine, and gadoterate meglumine at equimolar dose. Invest Radiol. 2013;48:121-128.

20. Investigators UJ, Morita A, Kirino T, Hashi K, Aoki N, Fukuhara S, Hashimoto N, Nakayama T, Sakai M, Teramoto A, Tominari S, Yoshimoto T. The natural course of unruptured cerebral aneurysms in a Japanese cohort. $N$ Engl $J$ Med. 2012;366:2474-2482. 
21. Tominari S, Morita A, Ishibashi T, Yamazaki T, Takao H, Murayama Y, Sonobe M, Yonekura M, Saito N, Shiokawa Y, Date I, Tominaga T, Nozaki K, Houkin K, Miyamoto S, Kirino T, Hashi K, Nakayama T, Unruptured Cerebral Aneurysm Study Japan I. Prediction model for 3-year rupture risk of unruptured cerebral aneurysms in Japanese patients. Ann Neurol. 2015;77:1050-1059.

22. Wiebers DO, Whisnant JP, Huston J, 3rd, Meissner I, Brown RD, Jr., Piepgras DG, Forbes GS, Thielen K, Nichols D, O'Fallon WM, Peacock J, Jaeger L, Kassell NF, Kongable-Beckman GL, Torner JC, International Study of Unruptured Intracranial Aneurysms I. Unruptured intracranial aneurysms: natural history, clinical outcome, and risks of surgical and endovascular treatment. Lancet. 2003;362:103110 .

23. Sonobe M, Yamazaki T, Yonekura M, Kikuchi H. Small unruptured intracranial aneurysm verification study: SUAVe study, Japan. Stroke. 2010;41:1969-1977.

24. Beck A, Teboulle M. A fast iterative shrinkage-thresholding algorithm for linear inverse problems. SIAM Journal on Imaging Sciences. 2009;2:183-202.

25. Becker S, Bobin J, Candès EJ. NESTA: a fast and accurate first-order method for sparse recovery. SIAM Journal on Imaging Sciences. 2011;4:1-39. 
Table 1. Patient demographics and characteristics.

\begin{tabular}{|c|c|c|c|}
\hline Patient & $\begin{array}{c}\text { Age } \\
\text { (years) }\end{array}$ & Sex & Location \\
\hline 1 & 38 & $\mathrm{M}$ & rt. VA \\
\hline 2 & 73 & $\mathrm{~F}$ & lt. ICA \\
\hline 3 & 72 & $\mathrm{~F}$ & lt. MCA \\
\hline 4 & 66 & $\mathrm{~F}$ & rt. MCA \\
\hline 5 & 46 & $\mathrm{M}$ & rt. MCA \\
\hline 6 & 74 & $\mathrm{~F}$ & lt. IC-PC \\
\hline 7 & 71 & $\mathrm{~F}$ & Acom \\
\hline 8 & 40 & $\mathrm{~F}$ & rt. ICA \\
\hline 9 & 72 & $\mathrm{~F}$ & lt. IC-PC \\
\hline 10 & 72 & $\mathrm{~F}$ & rt. ICA \\
\hline 11 & 38 & F & lt. ICA \\
\hline 12 & 62 & $\mathrm{~F}$ & rt. MCA \\
\hline 13 & 50 & F & rt. MCA \\
\hline 14 & 68 & M & Acom \\
\hline 15 & 74 & $\mathrm{~F}$ & lt. ICA \\
\hline
\end{tabular}




\begin{tabular}{|c|c|c|c|}
\hline 16 & 53 & $\mathrm{~F}$ & lt. ICA \\
\hline 17 & 73 & $\mathrm{~F}$ & lt. ICA \\
\hline 18 & 67 & $\mathrm{~F}$ & lt. ICA \\
\hline 19 & 76 & $\mathrm{~F}$ & Acom \\
\hline 20 & 70 & $\mathrm{~F}$ & lt. IC-PC \\
\hline $1^{*}$ & & & rt. MCA \\
\hline $6^{*}$ & & & lt. ICA \\
\hline $9 *$ & & & BA \\
\hline $15^{*}$ & & & rt. IC-PC \\
\hline $19 *$ & & & rt. MCA \\
\hline $20 *$ & & & rt. ICA \\
\hline
\end{tabular}

Note that the asterisk represents the patient who had a second aneurysm.

ICA, internal carotid artery; MCA, middle cerebral artery; IC-PC, internal carotidposterior communicating artery; Acom, anterior communicating artery; VA, vertebral artery; BA, basilar artery; rt, right; lt, left. 
Table 2. Measured neck, height and width of aneurysms.

\begin{tabular}{cccc} 
& Neck $(\mathrm{mm})$ & Width $(\mathrm{mm})$ & Height $(\mathrm{mm})$ \\
\hline PI-TOF 3× & $3.0[2.3-3.9]$ & $5.1[3.4-6.6]$ & $4.8[3.4-8.5]$ \\
Sparse-TOF 3× & $3.0[2.6-4.1]$ & $4.8[3.5-6.7]$ & $4.6[3.3-7.1]$ \\
Sparse-TOF 5× & $3.0[2.4-3.6]$ & $5.0[3.2-6.1]$ & $4.8[4.1-6.3]$
\end{tabular}

Note that the median [25th percentile - 75th percentile] of measured neck, height and width of aneurysms are shown. 


\section{Figure Legends}

Figure 1.

The sampling patterns of ky-kz phase-encoding plane are shown: PI-TOF 3× (left), Sparse-TOF 3× (middle) and Sparse-TOF 5× (right), respectively. Note the center part of k-space was fully sampled. Variable-density Poisson disc pattern was adopted for Sparse-TOF.

Figure 2

A 73-year-old female (Patient 2) with a huge aneurysm of the left internal carotid artery. Representative source images of PI-TOF 3× (a, f), Sparse-TOF 3× (b, c, g, and $h)$ and $5 \times(d, e, i$ and $j)$ are shown. The images (b, d, g and i) correspond to reconstruction with one iteration and the images (c, e, h and j) correspond to reconstruction with 10 iterations. There are some aliasing artifacts and blurring artifacts in the source image of Sparse-TOF with 1 iteration (b, d, g and i). Artifacts are reduced in the source images of Sparse-TOF with 10 iterations (c, e, h and j). Eventually, the interface between arteries and adjacent brain parenchyma or cerebrospinal fluid space became clearer in Sparse-TOF with 10 iterations (c, e, h and j). Artifacts in Sparse-TOF 
$3 \times$ with 10 iterations (c, h) are less than those in Sparse-TOF 5× with 10 iterations $(e, j)$, however, there is little difference among them. The noise in brain parenchyma is less in Sparse-TOF $3 \times$ with 10 iterations $(\mathrm{c}, \mathrm{h})$ than that in PI-TOF $3 \times(\mathrm{a}, \mathrm{f})$.

Figure 3

Maximum intensity projection (MIP) images of a 72-year-old female (Patient 3) with an aneurysm of the left middle cerebral artery (arrows). PI-TOF $3 \times$ in the upper row (a, b), Sparse-TOF $3 \times$ with 10 iterations in the middle row (c, d), and Sparse-TOF $5 \times$ with 10 iterations in the bottom row $(e, f)$. The aneurysm can be recognized well in all images.

Figure 4

Maximum intensity projection (MIP) images of 72-year-old female (Patient 9) with aneurysms of the left internal carotid-posterior communicating artery (arrows) and basilar artery (arrowheads). PI-TOF 3× in the upper row (a, b), Sparse-TOF $3 \times$ with 10 iterations in the middle row (c, d), and Sparse-TOF 5× with 10 iterations in the bottom row (e, f). The two aneurysms are recognized well in all images. 
Figure 5

Edge sharpness of an aneurysm of the left IC-PC aneurysm (arrow) (Patient

9) was calculated for the following images (a): PI-TOF $3 \times$ image, Sparse-TOF $3 \times$ and Sparse-TOF 5× images reconstructed with 1, 2, 3, 4, 5, 10, 15, 20, 30, 40 and 50

iterations (b). Zoomed images of (b) are also shown (c). The number of left bottom corner of each image of Sparse-TOF $3 \times$ and Sparse-TOF $5 \times$ represents the iteration number. Edge sharpness increased rapidly between 1 and 10 iterations; however, it did not increase any more after 20 iterations. Edge sharpness of Sparse-TOF 3× was largest, and that of Sparse-TOF $5 \times$ was second largest. Increase of iteration times require more post-imaging reconstruction time, 10 iterations adopted in this study may require minimum reconstruction time with adequate edge sharpness to recognize the edge of aneurysm.

Figure 6.

The original line profiles and fitted line profiles of the aneurysm and neighboring structure on the source image of PI-TOF $3 \times$ (left), Sparse-TOF $3 \times$ (middle) and Sparse-TOF 5× (right) are shown. The original line profile lacks in sampling point, therefore, curve fitting was performed to increase sampling point so as to obtain $20 \%$ 
and $80 \%$ of maximum signal intensity easily. Darker markers of Sparse-TOF $3 \times$ and Sparse-TOF $5 \times$ represent the fitted line profiles of Sparse-TOF $3 \times$ or Sparse-TOF $5 \times$ with fewer iterations. The original line profiles and fitted line profiles becomes sharper with more iteration times. 
Figure 1

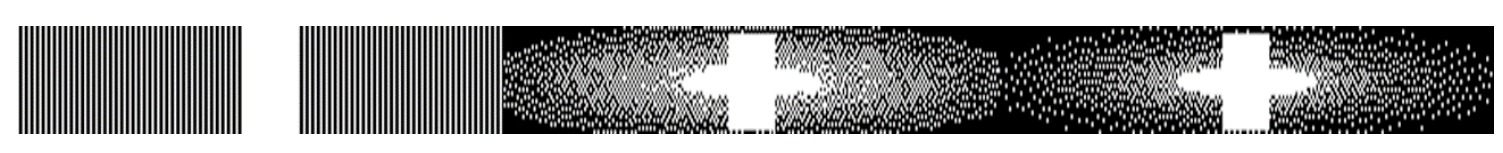


Figure 2

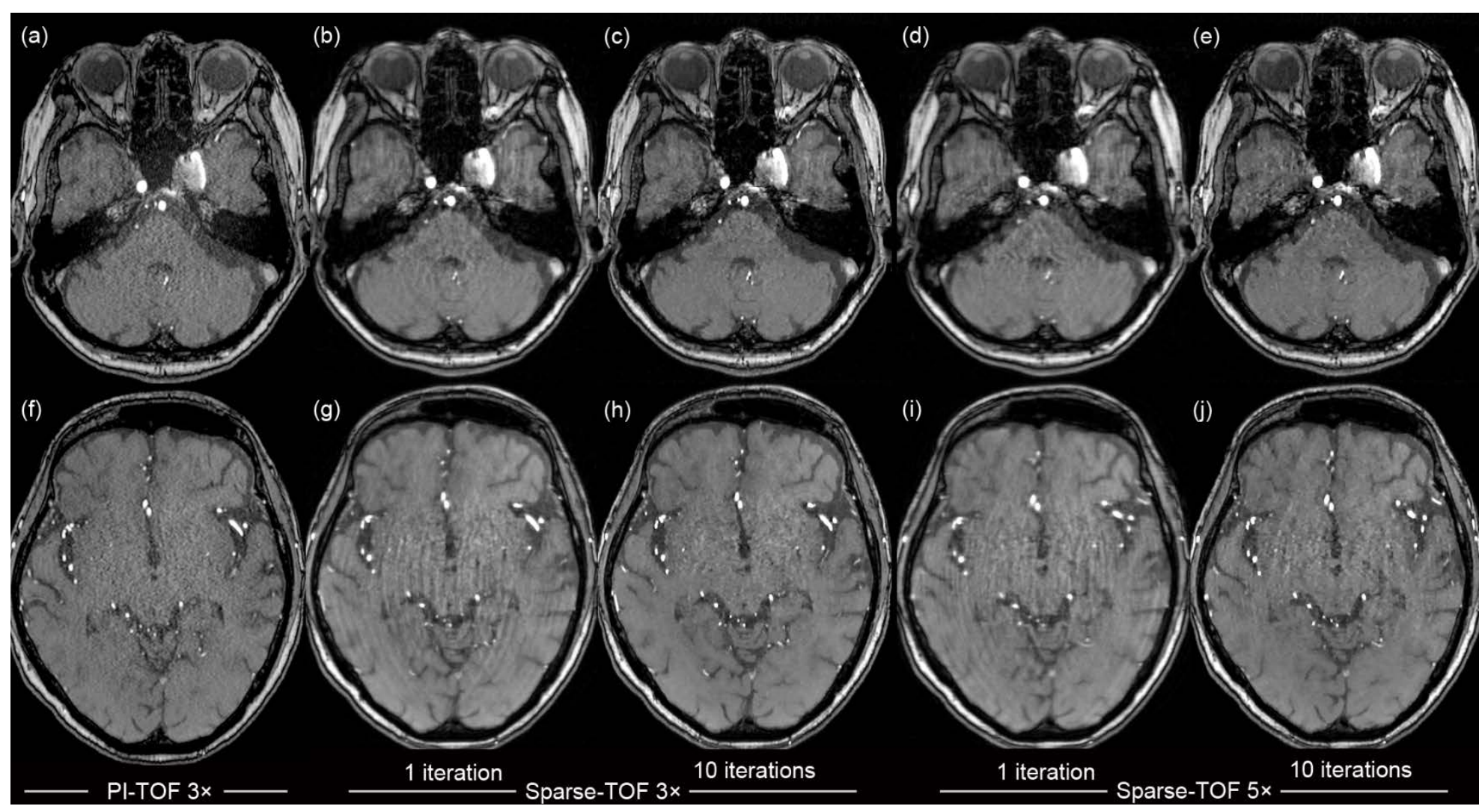




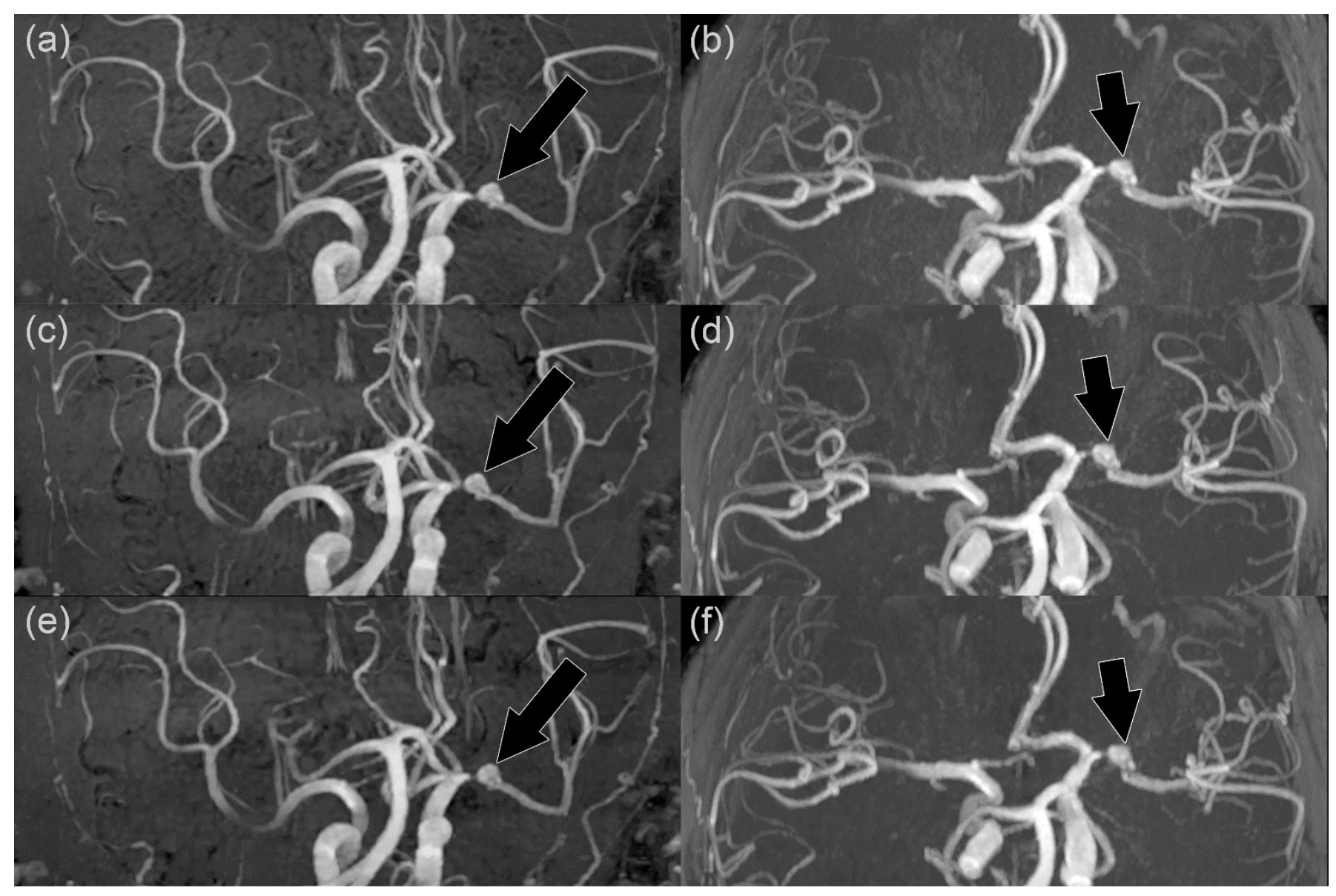


Figure 4

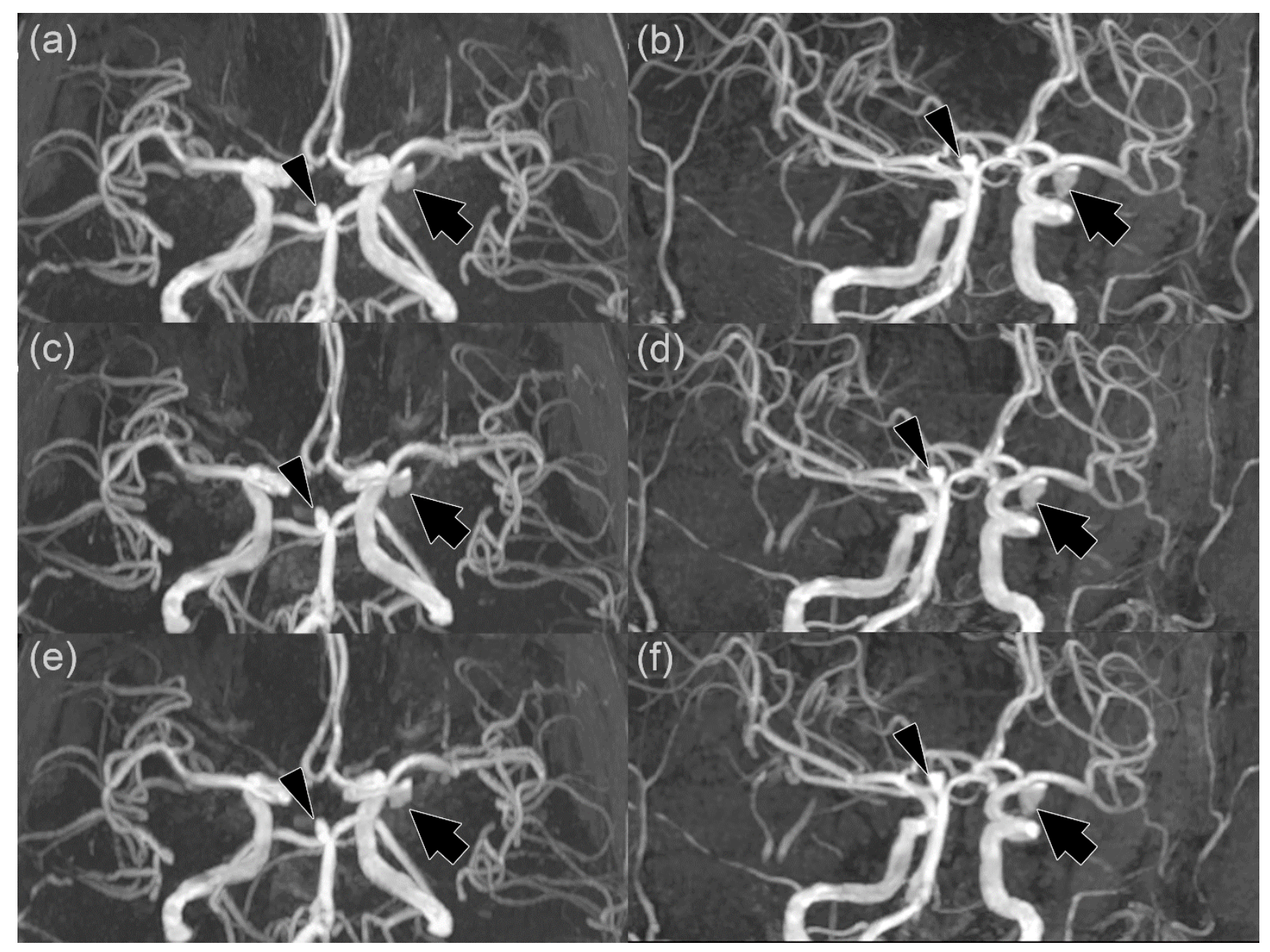


Figure 5(A)

\section{Edge Sharpness}

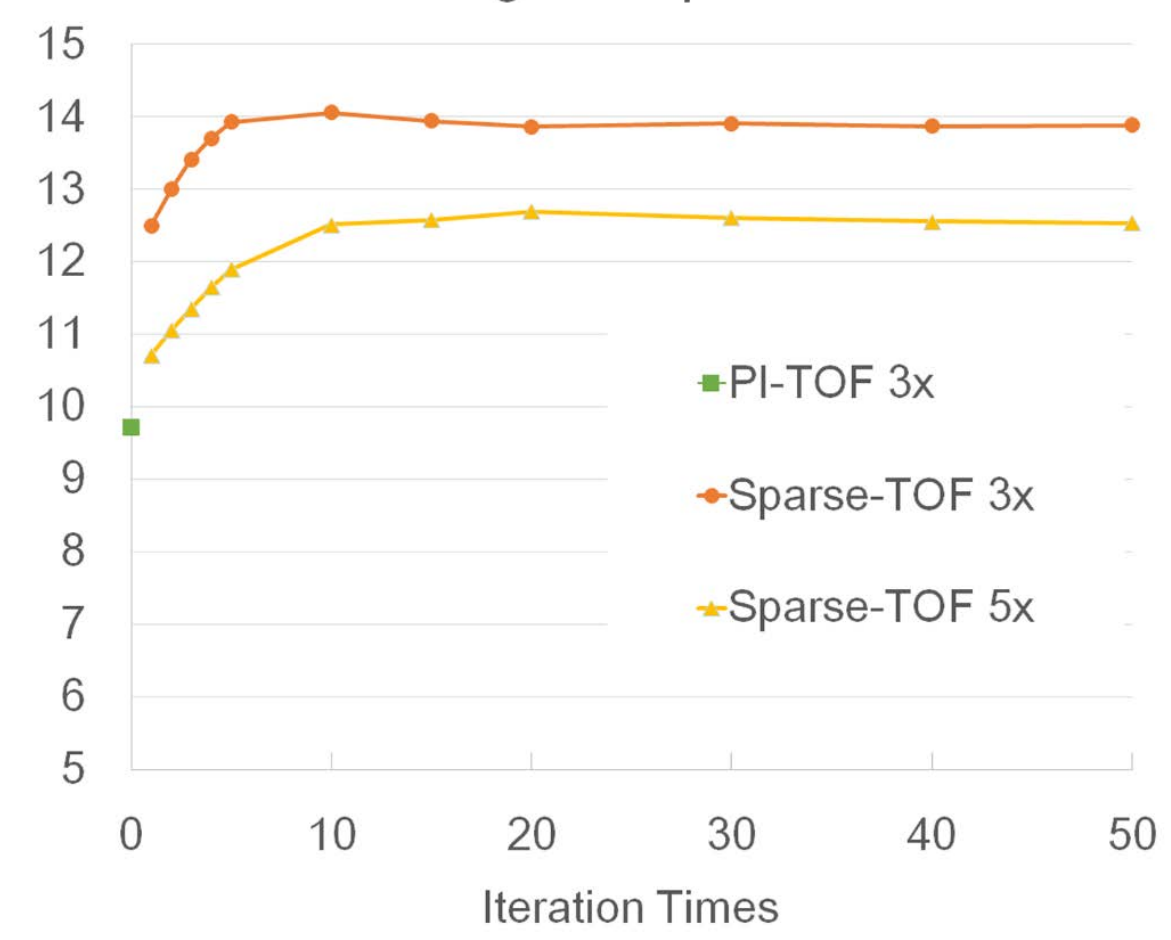


Figure 5(B)

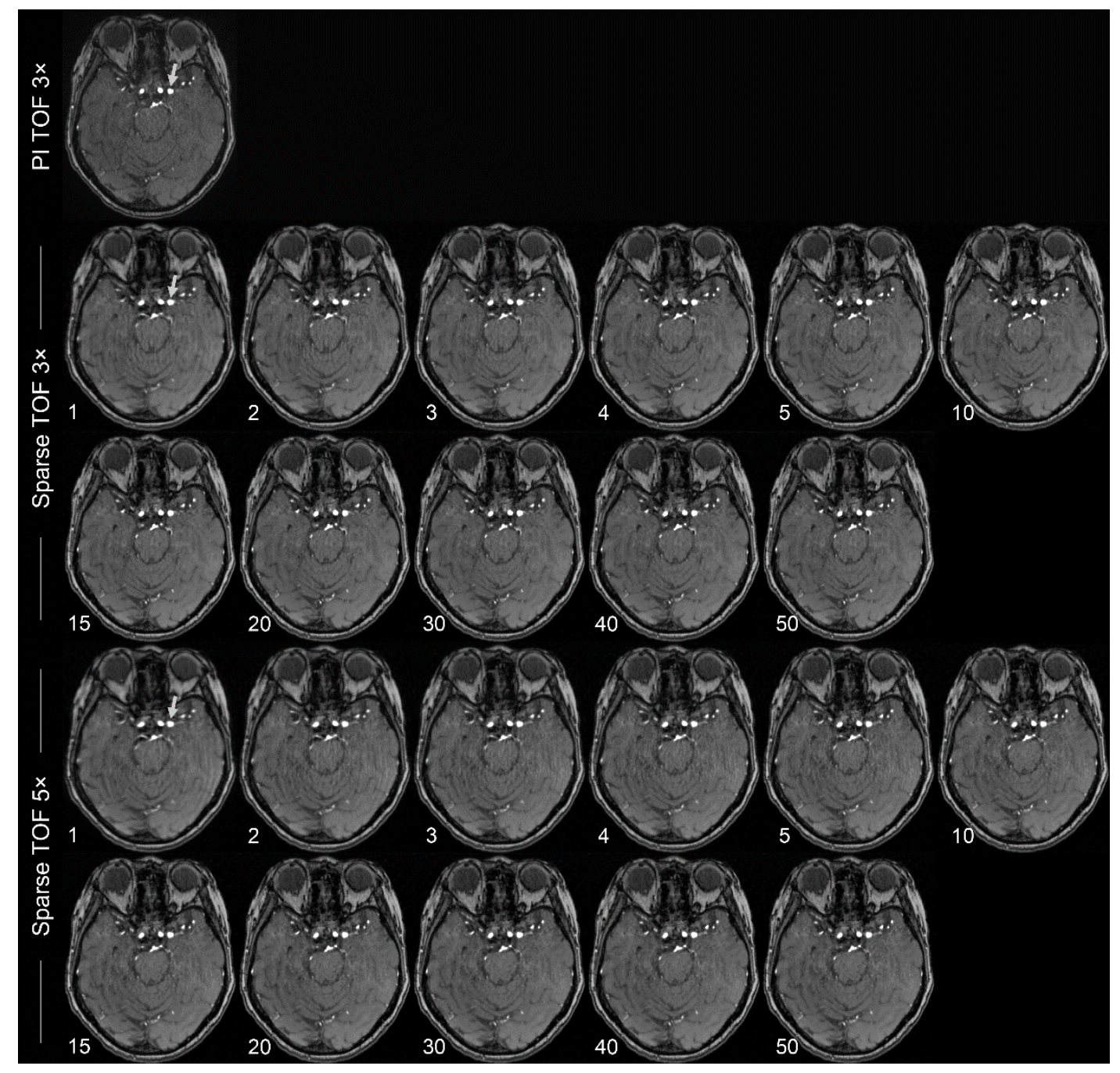


Figure 5(C)

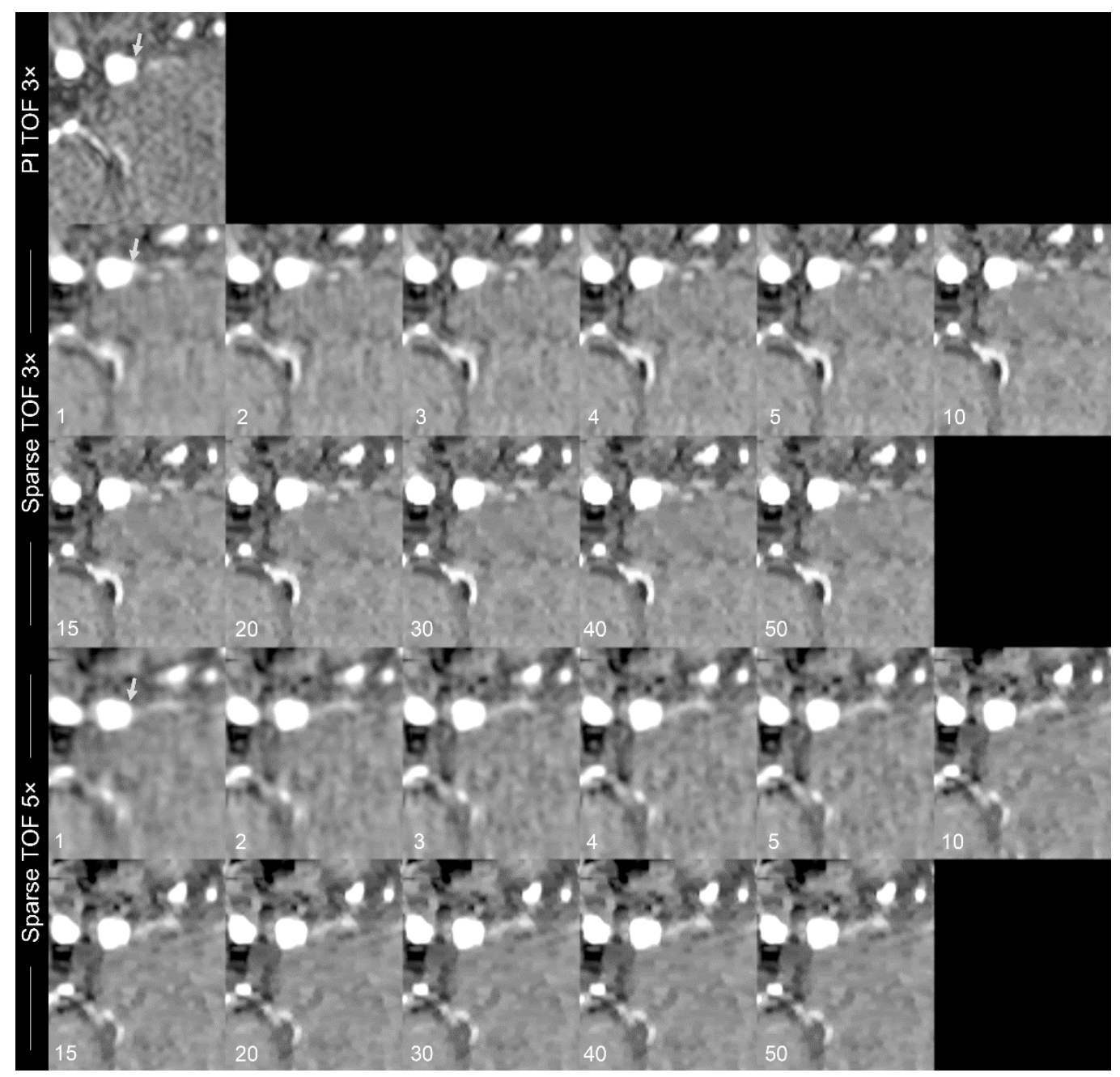


Figure 6
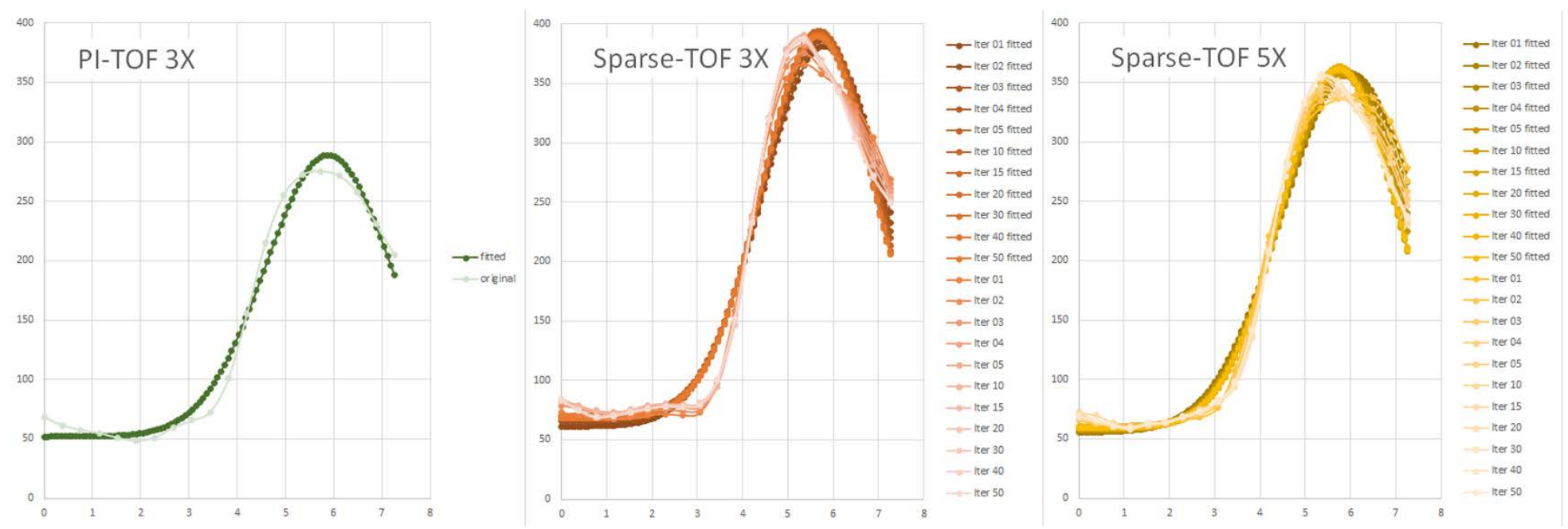